\title{
Efecto de la Adición de Edulcorantes no Calóricos sobre las Propiedades Fisicoquímicas y la Cinética de Secado de Cáscara de Mango Liofilizado
}

\author{
Liliana Serna-Cock ${ }^{(1) *}$, Cristian Torres-León ${ }^{(2)}$ y Alfredo Ayala-Aponte ${ }^{(3)}$ \\ (1) Univ. Nacional de Colombia, Fac. de Ing. y Admón., Sede Palmira. Carrera 32 Chapinero, \\ Vía Candelaria, Bloque 25, 3ํpiso, Palmira, Valle del Cauca-Colombia (e-mail: Iserna@unal.edu.co). \\ (2) Univ. Autónoma de Coahuila, Depto de Investigación en Alimentos. Blvd. Venustiano Carranza. Col. \\ República, 25280 Saltillo, Coahuila-México (e-mail: ctorresleon@uadec.edu.mx). \\ (3) Univ. del Valle, Escuela de Ing. de alimentos, 25360 Cali, Valle del Cauca -Colombia \\ (e-mail: alfredo.ayala@correounivalle.edu.co)
}

Recibido Dic. 17, 2014; Aceptado Feb. 17, 2015; Versión final Feb. 28, 2015, Publicado Ago. 2015

\begin{abstract}
Resumen
Se evaluó el efecto de la adición de edulcorantes no calóricos (estevia y aspartamo) sobre propiedades fisicoquímicas de cáscaras de mango liofilizadas (Variedades Kent y Keitt). Se midieron las cinéticas de secado y porosidad. Se midió actividad de agua, rehidratación, pH, acidez, y contenido de fibra. Los resultados mostraron que durante el secado, las muestras tratadas con los edulcorantes presentaron menores contenidos de humedad, porosidad y rehidratación que las muestras control. Los tratamientos con aspartame presentaron mayor porosidad y rehidratación, que con estevia $(p<0.05)$. Las dos variedades de cáscaras de mango presentaron altos contenidos de fibra dietaría total ( 17.8 para Kent y 22.4 para Keitt). Todos los tratamientos presentaron características de producto deshidratado estable con humedad menor a $7.09 \%$ (base seca) y actividad del agua menor a 0.37 . Cáscaras de mango deshidratadas y con solutos no calóricos podrían ser útiles para el consumo diario o aplicación en cereales.
\end{abstract}

\section{Effect of Adding Non-Caloric Sweeteners on the Physicochemical Properties and on the Drying Kinetics of Lyophilized Mango Peels}

\begin{abstract}
The effects of the addition of non-caloric sweeteners (stevia and aspartame) on the physicochemical properties of lyophilized mango peels (Kent and Keitt varieties) were evaluated. Drying and porosity kinetics were measured. Water activity, hydration, $\mathrm{pH}$, titratable acidity and fiber content were measured. The results showed that during drying, the samples treated with the two sweeteners, had lower contents of humidity, porosity and rehydration, compared to the untreated control samples. Treatments with added aspartame showed porosity and rehydration values higher than the samples treated with stevia $(p<0.05)$. The two varieties mango peels had high total dietary fiber content ( 17.8 for Ken and $\sim 22.4$ for Keitt). All treatments showed the uasua features of stable dehydrated product, with humidity lower than $7.09 \%$ (dry basis) and water activity lower than 0.37 . Dehydrated mango peels with non-caloric solutes can be used for direct consumption, or may be incorporated to cereals.
\end{abstract}




\section{INTRODUCCIÓN}

En Colombia se producen 221.015 Tm de mango al año (FAOSTAD, 2011), siendo las variedades florida las más usadas para el procesamiento de pulpa y consumo en fresco para exportación (Asofrucol, 2012). Además de la utilización de la pulpa, la cáscara se aprovecha en menor porcentaje principalmente para la fabricación de concentrados para animales. En general la cáscara de mango es un residuo poco aprovechable y contaminante del medio ambiente, por lo que es relevante valorarla para uso alimentario, debido a sus componentes nutricionales y bioactivos que varían de acuerdo a la variedad (Serna et al., 2015). De acuerdo a Ajila et al., (2007), las cascaras de mango son fuente de compuestos fotoquímicos como polifenoles, carotenoides, vitaminas, enzimas y fibra dietaría.

El consumo de fibra dietaría es importante para la nutrición humana y prevención de enfermedades, se recomienda el consumo de $38 \mathrm{~g} / \mathrm{d}$ para hombres entre 19 y 50 años, de $30 \mathrm{~g} / \mathrm{d}$ para hombres mayores de 50 años, de $25 \mathrm{~g} / \mathrm{d}$ para mujeres entre 19 y 50 años, y de $21 \mathrm{~g} / \mathrm{d}$ para mujeres mayores de 50 años (Grooms et al., 2013). Por lo anterior, cada vez se incrementa el consumo diario de alimentos con contenido de fibra, como en el caso de productos elaborados con cereales y frutos secos (Foschia et al., 2013). De otro lado, los edulcorantes bajos en calorias proporcionan sabor dulce con mínimo de calorias (Lemus et al. 2012) y son de amplio uso en el diseño de productos con bajo contenido calórico. La estevia es un edulcorante no calórico entre 160 a 220 veces más dulce que la sacarosa (American Dietetic, 2004), mientras que el aspartamo otro soluto no calórico es 300 veces más dulce que la sacarosa (Lemus et al., 2012).

El secado es uno de los métodos más antiguos de conservación de alimentos permitiendo menor susceptibilidad a la degradación microbiana, y permite reducir el volumen y peso lo que influye en una reducción importante de los costos de empaque, almacenamiento y transporte (Vega, et al., 2005). La liofilización es una técnica de secado apropiada para los alimentos, ya que no afecta la estabilidad y la actividad de compuestos bioactivos; además es un método de interés para la conservación de alimentos que permite prolongar el tiempo de vida útil y mantener significativamente propiedades físicas y fisicoquímicas relacionadas con la calidad (Ayala et al., 2010). Consiste en la eliminación del agua mediante dos etapas, primero se congela el alimento y posteriormente se elimina el hielo por sublimación desde la fase sólida hacia la fase vapor (Vasiliki et al., 2011). Los principales beneficios de la liofilización son la disminución de la actividad de agua $\left(\mathrm{a}_{\mathrm{w}}\right)$, mejoramiento de la textura y aumento de la porosidad producida por la sublimación de los cristales de hielo (Kasper et al., 2013). En este sentido, las cáscaras de frutas impregnadas con edulcorantes no calóricos y secadas, son potencialmente matrices alimentarias con contenido de fibra y otros nutrientes que podrían ser de consumo diario. Por consiguiente, el objetivo de este trabajo fue evaluar el efecto de la adición de edulcorantes no calóricos (estevia y aspartamo) sobre propiedades físicas y químicas de cáscaras de mango liofilizadas de las variedades Kent y keitt. Las variables de respuesta fueron las cinética de secado y de porosidad, aw, rehidratación, $\mathrm{pH}$, acidez titulable y contenido de fibra.

\section{MATERIALES Y MÉTODOS}

Materia prima: Se utilizaron mangos (Mangifera indica L.) de las variedades kent y keitt en estado de madurez 3 (ICONTEC, 2003). Los frutos fueron proporcionados por la empresa frutícola Barahonda del municipio de Palmira, Departamento del Valle del Cauca (Colombia). Se emplearon 65 mangos de la variedad keitt (peso por unidad de 507,63 g $\pm 31,01$ ) y 63 mangos de la variedad kent (peso por unidad de $562,43 \mathrm{~g} \pm 23,41$ ). Los frutos se lavaron con agua potable y se sumergieron en agua clorada (100 ppm de hipoclorito de sodio) durante $10 \mathrm{~min}$. Posteriormente, se les retiró la cáscara mediante el uso de un pelador manual y un cuchillo en acero inoxidable previamente desinfectados (200 ppm).

Impregnación de edulcorantes no calóricos y proceso de liofilización: Las cáscaras de mango de las dos variedades se cortaron en círculos de $15 \mathrm{~mm}$ de diámetro y $2 \mathrm{~mm}$ de espesor, utilizando un sacabocados en acero inoxidable previamente desinfectado con hipoclorito de sodio (200 ppm). Las cascaras se sumergieron en hipoclorito de sodio $(10 \mathrm{ppm})$ durante $1 \mathrm{~min}$, se enjuagaron con agua potable y se dejaron escurrir por $2 \mathrm{~min}$. Las muestras de cada variedad se dividieron en tres grupos: el primer grupo se sumergió en una solución de estevia (EST) comercial al $1.5 \%$, el segundo se sumergió en una solución de aspartamo (ASP) comercial al $2 \%$, utilizando $20 \mathrm{~g}$ de edulcorante por litro de agua, y el tercer grupo corresponde a las cascaras de mango sin tratamiento (CM). El proceso de inmersión de las muestras se realizó durante 35 min a $25^{\circ} \mathrm{C}$ con agitación. La relación entre la cáscara y el medio líquido fue de 1:4. Las muestras se retiraron de cada solución eliminando rápidamente la solución remanente de la superficie con agua destilada. Para el proceso de liofilización, las muestras se congelaron a $-30^{\circ} \mathrm{C}$ y se liofilizaron a presión de vacío de $8 \mathrm{~Pa}$ en un equipo (LABCONCO Freezone 6, USA). Las medidas de contenido de humedad (C.H), para obtener las cinéticas de secado y la porosidad se realizaron a diferentes tiempos del proceso de liofilización $(0,4,8,10$, $12,13$ y $14 \mathrm{~h})$. La aw, $\mathrm{pH}$, y acidez titulable se determinaron al inicio $(0 \mathrm{~h})$ y al final de secado (14 h). La capacidad de rehidratación y el contenido de fibra se midieron al final de la liofilización. 
Cinéticas de secado: El $\mathrm{CH}$ se midió a diferentes tiempos de liofilización, (0, 4, 8, 10, 12,13 y 14 h), utilizando una balanza de humedad infrarroja (Mettler Toledo AR-204, SUIZA), previamente calibrada para el producto específico. Con los valores de humedad se construyeron las curvas de humedad (b.s) vs. Tiempo (h).

Cinéticas de Porosidad $(\mathcal{E})$ : La $\varepsilon$ se determinó en los mismos tiempos de las cinéticas de secado, mediante la relación de la densidad aparente $\left(\boldsymbol{\rho}_{\mathrm{a}}\right)$ y la densidad real $\left(\boldsymbol{\rho}_{\mathbf{r}}\right)$ (Ec. 3). La $\boldsymbol{\rho}_{\mathrm{a}}$, se determinó por el método de deslazamiento de volumen con un picnómetro a $20^{\circ} \mathrm{C}$, y se calculó con la ecuación 1 (Alain, 2004). La $\rho_{\mathbf{r}}$ se estimó con la ecuación 2 (Lewis 1993).

$$
\begin{aligned}
& \rho a=\frac{P_{g}}{\frac{\left(P_{p+f}-P_{p}\right)-\left(P_{p+g+f}-P_{p+g}\right)}{\rho_{f}}} \\
& \rho r=\frac{1}{\frac{X w}{1000}+\frac{1-X w}{1590}} \\
& \varepsilon=\left(1-\frac{\rho_{a}}{\rho_{r}}\right) * 100
\end{aligned}
$$

En estas ecuaciones, $P_{g}$ es el peso de la muestra, $P_{p}$ es el peso del picnómetro, $P_{p+f:}$ es el peso del

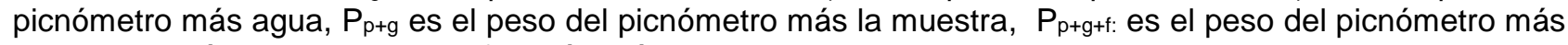
la muestra más agua y $\mathrm{X}_{\mathrm{w}}$ : es la fracción másica de agua en la muestra.

Actividad de agua: Las medidas de aw se midieron $25^{\circ} \mathrm{C}$ mediante un Aqua Lab (Decagon, 3TE, USA), Previamente calibrado con sales de actividades de agua entre 0,25 y 0.9. Las aw se midieron a muestras frescas $(\mathrm{t}=0 \mathrm{~h})$ y al final del secado $(14 \mathrm{~h})$.

Rehidratación: La rehidratación de las muestras de cáscaras de mango se estimó de acuerdo a la metodología descrita por Ayala et al., (2010); para ello se sumergieron las muestras liofilizadas (correspondientes al final del secado) en recipientes que contenían $25 \mathrm{ml}$ de agua destilada, la relación en peso de la muestra - agua destilada fue de 1:15. Se retiraron muestras de los recipientes en diferentes tiempos hasta alcanzar 180 min, se secaron con papel adsorbente y se calculó su peso y su $\mathrm{CH}$.

Acidez titulable y pH: La acidez se determinó por titulación con solución de $\mathrm{NaOH} 1 \mathrm{M}$ a pH 8,1 (usando un pH-metro (Mettler Toledo, Suiza). Se expresó como \% de ácido cítrico en base seca según lo descrito por la NTC 4623 (ICONTEC 1999b). El pH se midió con un pH-metro digital (Mettler Toledo, Suiza).

Contenido de fibra: El contenido de fibra en cáscaras liofilizadas se determinó por el método de Van Soest et al., (1991), identificando el contenido porcentual en base seca de hemicelulosa, celulosa y lignina presente en el material. La fibra dietética se determinó de acuerdo al método AOAC 991.43.

Diseño estadístico: Se utilizó un diseño multifactorial al azar de $2^{2}$. Como primer factor o variable de proceso fue la variedad de cáscara de mango, con dos niveles, variedad Kent y variedad Keitt, el segundo factor fue el edulcorante no calórico, con dos niveles EST y ASP. Los valores experimentales se presentaron como media \pm Desviación estándar. Cada tratamiento se realizó por triplicado. Los resultados se analizaron mediante análisis de varianza (ANOVA) con nivel de confiabilidad de 95\%, para ello se empleó el programa SAS (SAS 9.3).

\section{RESULTADOS Y DISCUSIÓN}

Los valores de C.H, aw, y porosidad en las cascaras frescas para las variedades Kent fueron respectivamente, $3,25 \pm 0.09 \mathrm{~g}$ agua $/ \mathrm{g} \mathrm{m.s,} 0.8356 \pm 0.0024$ y $4.48 \pm 0.07 \%$, mientras que para keitt fueron $4.05 \pm 0.03 \mathrm{~g}$ agua/g m.s, $0.8486 \pm 0.0007$ y $3.48 \pm 0.05 \%$

\section{Curvas de secado}

En la figura 1, se presentan las curvas de secado de las cáscaras de mango liofilizadas sin tratamiento (CM) y las tratadas con edulcorante ASP y EST. La figura (a) corresponde a la variedad Kent y la (b) a Keitt. Las muestras tratadas con solutos edulcorantes en ambas variedades mostraron menores cinéticas de secado respecto a las no tratadas. Este comportamiento se debe posiblemente a la formación de un encostramiento o capa de solutos en la superficie de las cascaras ocurrido durante la impregnación, provocando resistencia 
a la salida del agua libre durante el secado (Prothon, et al., 2001). El ANOVA evidenció un efecto significativo $(p<0.05)$ del tipo de tratamiento sobre el $\mathrm{C} . \mathrm{H}$ de las muestras, excepto en el tiempo $14 \mathrm{~h}$. A pesar que las curvas de secado en todos los tratamientos se realizaron a $14 \mathrm{~h}$, se puede considerar $12 \mathrm{~h}$ como el tiempo de secado final por los bajos niveles de agua alcanzados entre 12.9 y $6.8 \%$ b.s. En general los tres tratamientos de la variedad Kent para un mismo tiempo de secado mostraron ligeramente mayores pérdidas de humedad respecto a los de Keitt. Este resultado puede estar influenciado, por un lado por la mayor porosidad inicial de la cascara $(4.48 \pm 0.07 \%)$ permitiendo mayor transporte del agua, y por otro lado también por contener menor humedad en cada tratamiento al inicio del proceso (3.25 \pm 0.09 para CF, $3.33 \pm 0.17$ para ASP y $3.34 \pm 0.46 \mathrm{~g}$ agua/g m.s para EST). Al comparar las cinéticas entre los tratamientos con solutos no calóricos, se evidencia en las dos variedades que las muestras impregnadas con ASP mostraron mayores pérdidas de agua durante el proceso de liofilización, debido posiblemente a que estas moléculas se difunden más fácilmente a través de la matriz celular de las cascaras de mango, dado a que su peso molecular de 294,3 g/mol es menor (Guerrero, et a., 2014) que la de estevia con $804.80 \mathrm{~g} / \mathrm{mol}$ (García et al., 2009). Al difundirse el soluto se genera gradientes de concentración en la matriz celular, permitiendo así extraer más agua desde el interior de la célula.

(a)

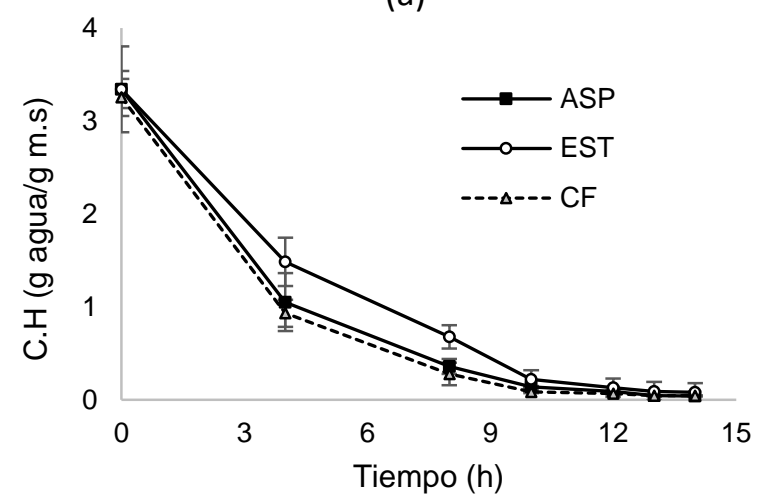

(b)

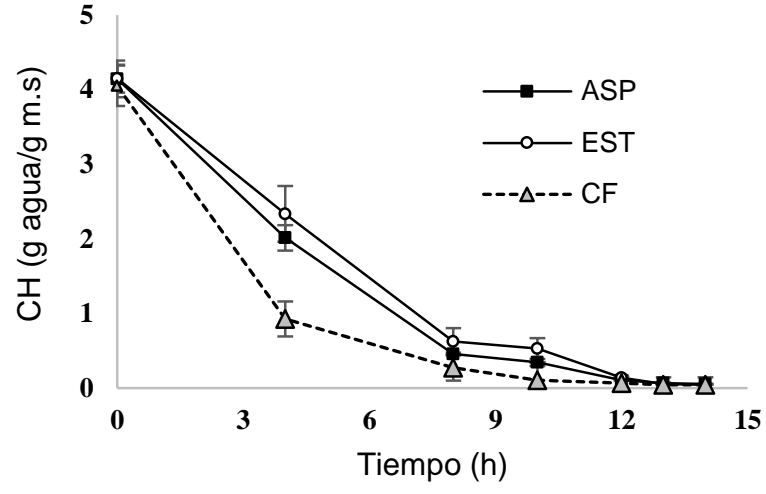

Fig. 1. Curva de secado de cáscaras de mango. Variedad Kent (a) y Variedad Keitt (b) tratadas con edulcorante Aspartamo (ASP), estevia (EST) y sin tratamiento (CML).

\section{Porosidad}

En la figura 2, se presentan los cambios de $\varepsilon$ de las muestras de cáscara de mango durante el proceso de liofilización. En ambas variedades de mango, las cáscaras presentaron bajos valores $\varepsilon$ en estado fresco con $4.48 \pm 0.07 \%$ para Kent y $3,48 \%$ para Keitt. El Análisis de varianza presentó un efecto significativo $(p<0,05)$ del tipo de variedad y de tratamiento sobre la $\varepsilon$. En todos los tratamientos, al inicio del proceso la $\varepsilon$ aumenta a una velocidad lenta, pero después de $8 \mathrm{~h}$ se incrementa rápidamente; similar comportamiento se ha observado en alimentos deshidratados por liofilización (Ayala, et al., 2010) y en otros métodos de secado (Aprajeeta, et al., 2015). Este incremento de la porosidad de las cascaras durante la liofilización puede ser atribuido a la sustitución del agua extraída en el secado por el aire dentro de los poros (Aprajeeta, et al., 2015). Por otro lado, a pesar que en este trabajo no se investigó el fenómeno de transición vítrea (Tg), este es un factor importante para explicar el incremento de la porosidad en productos liofilizados; Rahman, 2001 aduce que el incremento de la porosidad en alimentos liofilizados se debe a que la temperatura de congelación empleada es inferior a la de su Tg, manteniendo el producto en estado vítreo y sin cambios significativos en el volumen durante la liofilización.

Puede notarse, que las cascaras sin tratamiento (CF) en ambas variedades, presentaron significativamente mayores valores de $\varepsilon$ respecto a los tratamientos ASP Y EST, alcanzando al final del secado para Kent y Keitt valores de $74.32 \pm 0.42$ y $71.53 \pm 2.53 \%$ respectivamente, mientras que las muestras tratadas variaron entre 58.93 y $63.29 \%$. Este mismo comportamiento se presentó en los resultados de las curvas de secado, donde las muestras no tratadas mostraron mayor pérdida de agua, lo que indica que la porosidad está fuertemente relacionada con el contenido de humedad y de las condiciones de secado (Aprajeeta, et al., 2015). Por otro lado, en las muestras tratadas el proceso de impregnación de solutos en la matriz celular pudo haber ocasionado un debilitamiento o colapso de la estructura, y por consiguiente una disminución del tamaño del poro. 
(a)

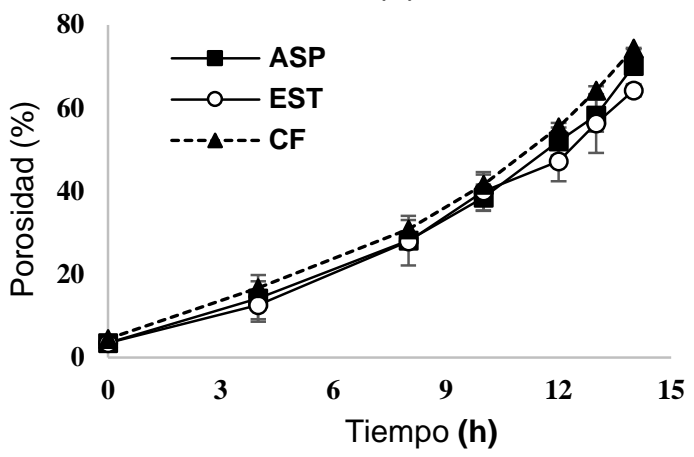

(b)

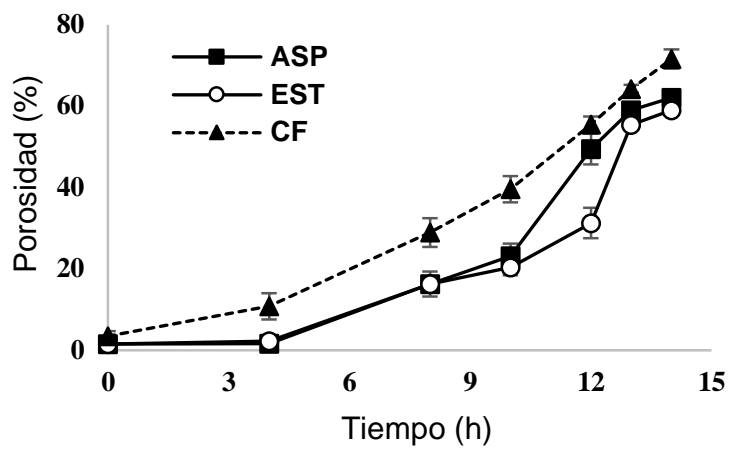

Fig. 2. Evolución de la porosidad en cáscaras de mango liofilizadas. Variedad Kent (a) y Variedad Keitt (b). Tratadas con edulcorante Aspartamo (ASP), estevia (EST) y sin tratamiento (CML).

\section{Actividad de agua}

En la tabla 1 se muestran los valores de $a_{w}$ de los diferentes tratamientos liofilizados. Estos valores fueron medidos al inicio y al final del secado, notándose que todos los tratamientos alcanzaron valores inferiores a 0.3763, lo cual significa que son seguros contra microorganismos durante el almacenamiento (Prabhakar and Mallika 2014). De acuerdo a Sing y Helman (1993), los alimentos con aw inferiores a 0,4 son seguros en el almacenamiento contra microrganismos y reacciones de oxidación, hidrolíticas, y actividad enzimática. Ross y Nichols (2014), manifiestan que la disminución de la aw es proporcional a la disminuir de la actividad microbiana. El ANOVA no mostró diferencias significativas $(P>0.05)$ al final del secado.

Tabla 1. Valores de actividad de agua en muestras de cáscara de mango fresca y tratada con solutos calóricos al inicio y final del secado por liofilización.

\begin{tabular}{|c|c|c|c|}
\hline \multirow[t]{2}{*}{ Tratamiento } & \multirow[t]{2}{*}{ Tiempo secado } & \multicolumn{2}{|c|}{ Variedad } \\
\hline & & Kent & Keitt \\
\hline CF & & $0.8356 \pm 0.0024$ & $0.8486 \pm 0.0007$ \\
\hline ASP & $0 \mathrm{~h}$ (Fresco) & $0.8423 \pm 0.0012$ & $0.8601 \pm 0.0002$ \\
\hline EST & & $0.8559 \pm 0.0003$ & $0.8684 \pm 0.0003$ \\
\hline CF & & $0.3566 \pm 0.0012$ & $0.3599 \pm 0.0007$ \\
\hline ASP & $14 \mathrm{~h}$ (Seco) & $0.3671 \pm 0.0014$ & $0.3614 \pm 0.0002$ \\
\hline EST & & $0.3763 \pm 0.0022$ & $0.3646 \pm 0.0003$ \\
\hline
\end{tabular}

\section{Rehidratación}

La figura 3 evidencia en todos los tratamientos, el incremento de la rehidratación de las muestras liofilizadas con el tiempo de proceso, mostrando alta velocidad de rehidratación durante los 5 min, y disminuyendo a los 60 min hasta obtener un valor constante para cada tratamiento. Al final del proceso los tratamientos alcanzaron valores de $\mathrm{CH}$ que variaron entre 74.63 y $75.77 \%$, valores cercanos a los del $\mathrm{CH}$ inicial de las cascara frescas; lo que indica que el proceso de rehidratación es reversible, asociado con mínimos daños estructurales y encogimiento celular (krokida, et al., 2003). Resultados similares se encontraron en el proceso de liofilización de acerola (Marques et al., 2007) y pitahaya amarilla (Ayala et al., 2010). El ANOVA evidenció diferencias significativas $(\mathrm{P}<0.05)$ del nivel de rehidratación entre los tratamientos de ambas variedades hasta los $30 \mathrm{~min}$. En general durante el proceso, las cascaras sin tratamiento (CF) mostraron los mayores valores de porosidad, seguidas del tratamiento ASP; estos mayores valores de rehidratación de las muestras CF están asociados a su mayor porosidad.

De acuerdo a Krokida, et al., (2003), la capacidad de rehidratación en alimentos deshidratados está influenciada por varios factores, principalmente el nivel de porosidad; a mayor porosidad mayor es la capacidad de rehidratación del alimento. Con respecto al tipo de variedad, los tratamientos con Kent presentaron ligeramente mayores valores de porosidad. 
(a)

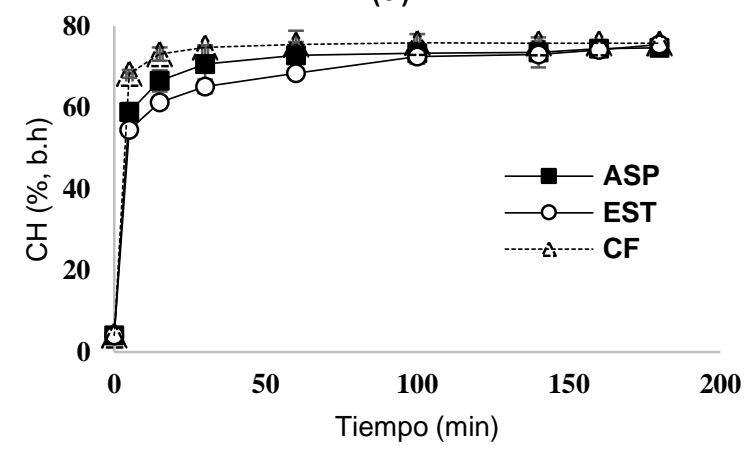

(b)

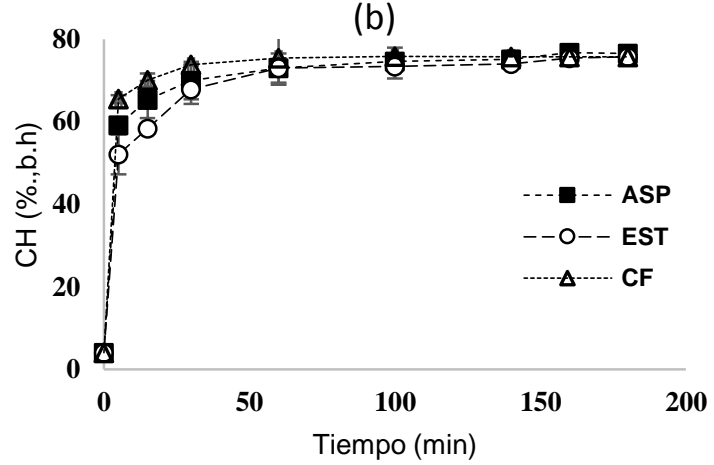

Fig. 3. Proceso de rehidratación de cáscaras de mango liofilizadas. Variedad Kent (a) y Variedad Keitt (b). Tratadas con edulcorante Aspartamo (ASP), estevia (EST) y sin tratamiento (CML).

\section{Acidez titulable y $\mathrm{pH}$}

EI ANOVA no mostró influencia significativas $(p<0.05)$ del tipo de variedad y del tratamiento sobre la acidez titulable al final del secado $(14 \mathrm{~h})$. Las muestras sin tratamiento alcanzaron $1.02 \pm 0.18$ para Kent y $0.92 \pm$ $0.17 \%$ para Keitt, mientras que las muestras Kent y Keitt tratadas con EST y ASP presentaron respectivamente $2.17 \pm 0.22,1.88 \pm 0.07,1.81 \pm 0.28$ y $1.88 \pm 0.20 \%$. Estos valores son relativamente

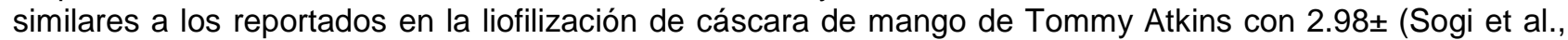
2013). Estos mismos autores argumentan que el método de liofilización retiene mayor acidez titulable en la cáscara de mango que otros métodos de secado; lo cual significa el efecto positivo de este método de secado. Con respecto al $\mathrm{pH}$, tampoco se evidenciaron diferencias significativas $(p<0,05)$ entre los tratamientos al final del secado; presentado las muestras sin tratamiento $5.12 \pm 0.22$ para Kent y $4.63 \pm 0.31$ para Keitt, mientras que para Kent y Keitt tratadas con EST y ASP fueron respectivamente 4,48 $\pm 0,026,4,69$ $0,05,4,19 \pm 0,17$ y $4,23 \pm 0,09$. Estos valores son similares a los reportados por Sogi et al. (2013) en cascaras de mango. El pH de las cáscaras de mango es un parámetro importante para determinar su utilización en productos sensibles al $\mathrm{pH}$. También como parámetro de estabilidad, ya que inhibe el crecimiento microbiano; valores bajos de $\mathrm{pH}$ garantizan la estabilidad de alimentos (Coton y Leguerinel 2014).

\section{Composición en fibra}

La tabla 2, muestra la composición de las cáscaras de mango de las variedades kent y keitt. Todos los valores son la media \pm DS de tres repeticiones. Se evidencia un alto contenido de materia seca en ambas variedades $(23,725 \pm 1,322$ y $18,406 \pm 0,008 \%)$, similar al reportado en cáscaras de la variedad Raspuri y Badami de la india (Ajila et al., 2007). Los contenidos de fibra dietética total (soluble e insoluble) fueron superiores en la variedad keitt con un $22,378 \pm 0,278 \%$, valor similar a los reportados en cascara de mango Tommy Atkins procedente de México (Vergara et al., 2007). Estos resultados de contenido de fibra dietética en las cácaras de las dos variedades de mango se consideran altos, ya que son superiores a los productos comerciales considerados de alto contenido en fibra dietética como el pan integral de centeno ( $\mathrm{FDI}=7,8 \%$; $\mathrm{FDS}=2,6 \%$; FDT $=10,4 \%$ ) y el pan integral de trigo (FDI = 5,8\%; FDS =1,4\%; FDT=7,2\%) (Hollmann et al., 2013). Con respecto al contenido de hemicelulosa y lignina, la variedad Keitt presentó los mayores contenidos, mientras que el contenido de celulosa fue superior en Kent.

Tabla 2. Composición (\% b.s.) de las cáscaras de mango liofilizadas de las variedades Kent y Keitt.

\begin{tabular}{|l|l|l|}
\hline Componente & Kent & Keitt \\
\hline Ms total & $23,725 \pm 1,322$ & $18,406 \pm 0,008$ \\
\hline FDS & $1,675 \pm 0,078$ & $4,203 \pm 0,978$ \\
\hline FDI & $16,140 \pm 0,240$ & $18,174 \pm 0,700$ \\
\hline FDT & $17,810 \pm 0,250$ & $22,378 \pm 0,278$ \\
\hline Lignina & $2,755 \pm 0,177$ & $4,708 \pm 0,649$ \\
\hline Hemicelulosa & $3,765 \pm 0,346$ & $3,835 \pm 0,449$ \\
\hline Celulosa & $8,550 \pm 0,346$ & $7,791 \pm 0,073$ \\
\hline
\end{tabular}




\section{CONCLUSIONES}

La adición de solutos no calóricos (aspartame y stevia) y la variedad (Kent y Keitt) afectaron significativamente propiedades físicas en cascaras de mango durante la liofilización. Las muestras tratadas con solutos no calóricos presentaron menores cinéticas de secado, porosidad y rehidratación respecto a las muestras control (no tratadas), siendo las muestras con adición de estevia las que presentaron los menores valores. Sin embargo, al final del proceso de secado tanto los tratamientos con adición de solutos no calóricos, como los tratamientos control, alcanzaron condiciones apropiadas de calidad y conservación de producto deshidratado, con contenidos de humedad y actividades de agua inferiores al $7.9 \%$ (b.s) y 0.37 respectivamente, valores de porosidad que variaron entre 58.93 y $74.32 \%$ y alta rehidratación. Por lo anterior la aplicación de edulcorantes no calóricos a cáscaras de mango liofilizadas sería una excelente alternativa de producto de alto contenido en fibra y de bajas calorías para ser incorporado a cereales para el consumo humano, o para el desarrollo de nuevos ingredientes y productos alimentarios.

\section{REFERENCIAS}

AOAC 991.43. Official methods of analysis of the Association of Oficial Analytical Chemists international. Methods of analysis for nutrion la beling chapter method 991.43.dietary fiber. Washington DC, Virginia: EUA. (1993)

Ajila, C., Bhat, S., Rao, P, Valuable components of raw and ripe peels from two Indian mango varieties. Food Chemistry: 102 (4), 1006-1011. (2007)

Alain, R. Reología y Análisis de La Textura de los Alimentos. Primera edición. Editorial Acribia S.A. Zaragoza, España (2004)

Ayala A., Serna L., Mosquera E. Liofilización de pitahaya amarilla ( Selenicereus Megalanthus ). Revista de La Facultad de Química Farmacéuitica: Univ.de Antioquia, Medellín, Colombia. 17 (100), 121-27 (2010)

Coton, E. Ecology of bacteria and fungi in foods. Effects of pH. Encyclopedia of Food Microbiology (Second Edition). Editorial Zoey Ayres, Simon Holt. 577-86 (2014)

Foschia, M., Peressini, D., Sensidoni A., Brennan, C. The Effects of Dietary Fibre Addition on the Quality of Common Cereal Products. Journal of Cereal Science: 58 (2) 216-27 (2013)

Grooms, K., Ommerborn M., Pham, D., Djoussé, L., Clark, C. Dietary Fiber Intake and Cardiometabolic Risks among US Adults, NHANES 1999-2010. The American J. of Medicine 126 (12) 1059-67 (2013)

García, P., Porlles L., Ráez, G., Romero, G. Rentabilidad del procesamiento de la stevia (stevia rebaudiana bertoni) como condición para ser considerada cultivo alternativo. Revista peruana de ingeniería química: 12 (1) $59-65$ (2009)

Hollmann, J., Themeier, H., Neese, U., Lindhauer, M. Dietary Fibre Fractions in Cereal Foods Measured by a New Integrated AOAC Method. Food Chemistry: 140 (3) 586-89 (2013)

ICONTEC 2003. Norma Técnica Colombiana 5210. Frustas frescas. Mango variedades mejoradas. Especificaciones (2003)

ICONTEC. 1999a. Norma Técnica Colombiana 4623. Productos de Frutas Y Verduras. Determinacion de La Acidez Titulable. (1999a)

Krokida, M y Marinos D. Rehydration kinetics of dehydrated products. Journal Food Engineering: 57 (1) 1-7 (2003)

Lemus, R., Vega, A., Zura, L., Ah, K. Stevia Rebaudiana Bertoni, Source of a High-Potency Natural Sweetener: A Comprehensive Review on the Biochemical, Nutritional and Functional Aspects. Food Chemistry: 132 (3) 1121-32 (2012)

Marques, L., Ferreira, M, Freire, T. Freeze-Drying of Acerola (Malpighia Glabra L.). Chemical Engineering and Processing: Process Intensification: 46 (5) 451-57 (2007) 
Prabhakar, K y Mallika, E. Water Activity. Encyclopedia of Food Microbiology (Second Edition). Editorial Zoey Ayres, Simon Holt. 751-54 (2014)

Ross, T., Nichols, D. Ecology of bacteria and fungi in foods. Influence of Available Water. Encyclopedia of Food Microbiology: 587-94 (2014)

Serna-Cock, L., Torres-León, C., Ayala- Aponte, A. Evaluación de polvos alimentarios obtenidos de cáscaras de mango (Mangifera indica) como fuente de ingredientes funcionales. Información Tecnologica 26(2), en prensa (2015)

Sogi, D., Siddiq, M., Greiby, I., Dolan, K. Total Phenolics, Antioxidant Activity, and Functional Properties of 'Tommy Atkins' Mango Peel and Kernel as Affected by Drying Methods. Food Chemistry: 141 (3) 2649-55 (2013)

Aprajeeta, J., Gopirajah, R., Anandharamakrishnan, S. porosity effects on heat and mass transfer during potato drying. Journal of Food Engineering: 144 119-128(2015)

Vega, A., Andrés, A., Fito, P. Modelado de la Cinética de Secado del Pimiento Rojo (Capsicum annuum L. cv Lamuyo). Información Tecnológica: 16 (6) 3-11(2005)

Van Soest, P., Robertson, J., Lewis, B. Methods for dietary fiber, neutral detergent fiber and nonstarch polysaccharides in relation to animal nutrition. J. Dairy Science: 74 3583-3597 (1991)

Vasiliki, P., Magdalini K. Vaios T. The influence of freeze drying conditions on microstructural changes of food products. Procedia Food Science: 1647 - 654. (2011)

Prothon, F., Ahrné, L., Funebo, T., Kidman, S., Langton, M., Sjöholm I. Effects of combined osmotic and microwave dehydration of apple on texture, microstructure and rehydration characteristics. Lebensm Wiss Technol: 34 (2) 95-101 (2001) 